\title{
A Qualitative Examination of a Mothers' Swim Program: What Keeps Them Coming Back and How Does it Improve Their Psychological Wellbeing?
}

\author{
Angela Huntsman ${ }^{1,2}$, John K Parker ${ }^{3}$ and Geoff $P$ Lovell ${ }^{*}$ \\ ${ }^{1}$ School of Social Sciences, University of the Sunshine Coast, Maroochydore, Australia \\ ${ }^{2}$ The Australian Institute of Human Development, Australia \\ ${ }^{3}$ School of Sport and Exercise, University of Gloucestershire, UK
}

*Corresponding author: Geoff P Lovell, PhD, School of Social Sciences, University of the Sunshine Coast, Locked bag 4, Maroochydore, QLD 4558, Australia, Tel: +61-(0)-5456-5100,E-mail: glovell@usc.edu.au

\begin{abstract}
Introduction: Exercise is widely accepted as an effective activity for alleviating the impact of psychosocial challenges associated with motherhood. However our current understanding of how to help mothers adhere to exercise and how it affects positive psychosocial outcomes is lacking.

Objective: To examine what aspects of a mother-focused swimming exercise program that participating mothers considered most influential in supporting their continuing exercise adherence as well as positively influencing their psychological wellbeing.

Methods: Qualitative thematic content analysis was performed on individual interview data from mothers, with a child of five years of age or younger, who regularly participated (three or more times a fortnight for six weeks or more) in the organized group based swim programs specifically for mothers $(\mathrm{N}=19)$.

Results: Mothers' perceptions of the factors supporting their adherence to the swim program were inherently intertwined with factors they considered positively influenced their mental health. Mothers' experiences of the swim program highlighted three higher order themes associated with supporting continued participation and positive psychological outcomes: Environmental factors; social support factors; and health promoting factors.

Conclusion: Environmental, social support, and health promoting factors are important factors in affecting the efficacy of mothers' exercise programs to enhance participation and wellbeing.
\end{abstract}

\section{Keywords}

Mothers, Exercise, Wellbeing, Adherence, Intervention

\section{Introduction}

Motherhood is a complex and challenging transition period for women and is associated with hormonal changes, actual and perceived negative changes in body image, and social changes including increased social isolation as well as loss of identity [1-5]. Motherhood is also reported to frequently be accompanied by role overload [6], weight retention [7], disturbance in sleep patterns [8], fatigue [9], and changes in daily routines [10]. With these changing roles and challenges, depression during and after pregnancy can be a major issue [11].

Exercise is widely accepted as an effective activity for enhancing psychological wellbeing [12-14] and mitigating the impact of psychosocial challenges faced by mothers $[9,15,16]$. Exercise can also be a cost effective treatment for poor mental health without harmful negative side-effects [14]. In a large sample of mothers from Australian and New Zealand, with the exception of anxiety, psychological wellbeing of mothers that reported exercising 3 to 4 times per week was significantly and meaningfully more positive in comparison to those mothers who reported not exercising [16]. Even only exercising once a week, compared to the non-exercising mothers, was associated with significantly lower depression scores [16].

Unfortunately, despite the reported diverse positive effects associated with exercise, motherhood rep-

Citation: Huntsman A, Parker JK, Lovell GP (2018) A Qualitative Examination of a Mothers' Swim Program: What Keeps Them Coming Back and How Does it Improve Their Psychological Wellbeing? Int J Womens Health Wellness 4:066. doi.org/10.23937/2474-1353/1510066 Received: November 19, 2015: Accepted: February 26, 2018: Published: February 28, 2018 Copyright: (c) 2018 Huntsman A, et al. This is an open-access article distributed under the terms of the Creative Commons Attribution License, which permits unrestricted use, distribution, and reproduction in any medium, provided the original author and source are credited. 
resents a major barrier to being physically active [17]. Previous research estimates that women with children exercise even less than women without children, of which two thirds do not exercise to an adequate level [18]. Ansari and Lovell [19] reported almost one third of women's perceived barrier to exercise was accounted for by the number of children she had. Furthermore, Albright, Maddock, and Nigg [20] observed only that 35\% of women who were active before childbirth were active after childbirth. This is despite Currie and Develin's [21] finding that $69 \%$ of women in the postnatal period indicated that they would like to exercise more.

The factors associated with why mothers generally do not engage in sufficient exercise is clearly complex with multiple personal, interpersonal, environmental, and policy determinants [22]. Furthering our understanding of what factors affect mothers' exercise behavior and how, has obvious potential to better inform exercise promotion interventions supporting positive public health outcomes; both physiological and psychological. However, while our understanding of antecedents of exercise behavior for general populations has substantially developed over the last two decades, our understanding for specific populations such as mothers remains limited [15].

While it is commonly accepted that exercise does protect and enhance mothers' psychological wellbeing [16], current theoretical explanations of this positive effect are limited [15]. Current explanations include biological, biochemical, and psychosocial mechanisms. Although understanding of this complex phenomenon is rapidly developing, awareness of the mechanisms by which exercise aids psychological wellbeing in specific populations, including mothers, remains especially limited. While there are sparse studies, for example Armstrong and Edwards [23], that have examined the effects of mother focused exercise programs, qualitative data examining the mothers' experience are exceptionally scant. In the same way that our restricted understanding of how and what factors support mothers' exercise participation limit the efficacious evidence based design of mothers' exercise interventions, so does our narrow understanding of how exercise aids psychological wellbeing limit the design of exercise interventions to best support positive mental health outcomes.

Self-Determination Theory (SDT) [24-26] and its sub-theory Basic Needs Theory (BNT) [27] was selected as the theoretical framework for this investigation. SDT and BNT contend that humans develop and function effectively as a consequence of the social environment and its potential to satisfy basic psychological needs. According to BNT, there are three basic human psychological needs: Autonomy; competence; and relatedness [24-27]. The inherent need for autonomy is satisfied when we perceive that we are the origin of our choices and decisions, and that we are acting in accordance with our integrated sense of self. Competence regards our need to feel a sense of mastery through effective interaction within our environment. The third need, relatedness, pertains to feeling securely attached to, and being respected by, significant others. Satisfaction of these psychological needs is considered to directly enhance motivation along with psychological and physical wellbeing. However, when basic psychological needs are thwarted, ill-being ensues [27]. Thus, if exercise is constructed in such a way such that it provides: A sense of control in terms of choice to participate and what that modality of exercise may be (autonomy); a perception of competence through generation of feedback related to positive progress towards goals (competence); and facilitates a sense of belonging through exercising in a group or with others (relatedness), motivation to exercise and psychological wellbeing should be enhanced.

Previous research has been successful in adopting SDT as a theoretical framework to examine motherhood and wellbeing $[28,29]$. Lovell, Gordon, Mueller, Mulgrew, and Sharman [29] found that mothers who exercised predominantly in group settings and those who exercised equally in group and individual contexts, experienced significantly higher satisfaction of autonomy and competence basic psychological needs as well as higher self-determined exercise motivation than those exercising predominantly in individual settings. The mothers exercising in individual contexts demonstrating the lowest levels of relatedness needs satisfaction.

\section{Aims}

In response to the limitations of previous research and set within the theoretical framework context of SDT, the objective of this investigation was to examine what aspects of a mother-focused swimming exercise program that participating mothers considered most influential in supporting their continuing exercise adherence, as well as positively influencing their psychological wellbeing. Specifically, two research questions were approached: Firstly, what are the salient factors most relevant to mothers participating in a mother-focused swimming exercise program in its support of sustained exercise adherence. Secondly, what are the factors of the swimming exercise program that participating mothers felt positively enhanced their psychological wellbeing.

In this project we defined mother as those with a child of five years of age or younger.

\section{Methods}

\section{Design}

This investigation used a descriptive, qualitative design.

\section{Recruitment and data collection}

Following institutional ethical approval (A/09/199), 
potential participants were recruited from two aquatic centers in Queensland Australia that held identical organized group based swim programs specifically for mothers with onsite childcare. Individuals were eligible for participation in this research if they had a child of five years of age or younger and that they regularly participated (three or more times a fortnight for six weeks or more) in the organized group based swim programs specifically for mothers. Recruitment involved fliers provided to all mothers taking part in the swim program at each site over a six week period. The fliers included a research participant information sheet. Mothers that wished to volunteer for the research did so by either contacting the data collecting researcher directly by phone, email, or post. From the 80 participants who volunteered, 20 were randomly selected for interview with 19 (95\%) completing the interviews. An initial target sample size of 20 was selected as a being the minimum number that would represent the swim program population. Upon analysis of the 19 participants, data were examined to assess whether data saturation had been achieved. As it was concluded that data saturation had been achieved with the initial sample of 20 , recruitment was not extended.

\section{The swim based exercise program}

The mothers' swimming program consisted of user-pays swim sessions held every Monday, Wednesday, and Friday mornings at 9:15 located at the two separate aquatic centers, each with different coaches yet run by the same organization. The start time was 9:15 am to allow mothers with older children to be dropped off prior to starting the swim session. The sessions were lane based swim training, led by an instructor who set each session and provide encouragement along with technical advice and feedback. The program was also accompanied by onsite childcare and café providing post-swimming social interaction opportunities for the mothers. The swim sessions lasted for approximately $1 \frac{1}{2}$ hours with typically between 20 and 35 mothers attending each session.

\section{Procedure and interviews}

Following informed consent to take part in the investigation, participants were placed in a pool of potential participants from which 20 were randomly drawn to be interviewed. Approximately one week before interview, participants completed a questionnaire including demographic measures. Interviews were conducted individually, mostly held at the aquatic centers due to the availability of childcare. Interviews lasted on average approximately 45 minutes.

Interviews followed a semi-structured format (see Figure 1 for an outline of the interview questions). Immediately following each recorded interview, the interviewer transcribed the content for later analysis.

\section{Data analysis}

Manifest content analysis (thematic) of the interview data followed the 5 processes detailed by Braun \& Clarke [30]: 1. Familiarizing with data set; 2. Gener-

1. Tell me about how you found this group?

2. What prompted you to seek out such a group?

3. How often do you come along to this swim program?

4. Is this your first foray into exercise since having children?

5. What kind of exercise programs were you participating in before you had children?

6. What is different about this group than say if you swam on your own?

7. Why do you come along to these sessions?

8. Does the onsite child care availability affect you?

9. If you met a mother, away from the pool context, would you suggest that she joined this mothers' swim program? Why?

10. With regard to the challenges of being a mother, how does this program help you?

11. Do you socialize with any of the other mothers after swimming or outside swimming? What sort of things do you do?

12. If you were not feeling up to swimming, do you ever still come along just to see the other mothers, even if you're not actually swimming? Why?

13. Have you made any friendships through this swim program; how would you describe them?

14. What would you say the five biggest positives of this program are for you?

Figure 1: Semi-structured interview schedule. 
ation of initial codes (plus validity checks); 3. Searching for themes; 4. Reviewing themes; and 5. Defining and naming themes. As part of the validity checks, an independent third party experienced in qualitative research but not involved in the research project reviewed the generated initial codes and proposed themes. As this research was informed by the SDT theoretical framework, higher order themes were conceptualized by this a priori consideration. However, initial codes and themes were not formed around a priori perspectives. The rationale for this process was to enable a flexible approach to the identification of themes that had importance and meaning to the research participants, yet allow for higher order themes to be contextualized within a theoretical perspective. Direct unidentified participants' quotes are presented to further illustrate and validate themes.

\section{Results}

\section{Participant descriptives and demographics}

The mean age of the 19 participants sample was 38.9 years $(S D=5.2)$. The mean age of the mothers at the birth of their first child was 29.2 years $(S D=5.2)$. Mean number of children was 2.6 (SD $=1.1$ ); $26 \%$ of the mothers had a youngest child under 1 year of age, $16 \%$ had a youngest child 1 year of age, $10 \%$ had a youngest child 2 years of age, $5 \%$ had a youngest child 3 years of age, $21 \%$ had a youngest child 4 years of age, and $21 \%$ had a youngest child 5 years of age. The majority of the mothers $(90 \%)$ reported their relationship status as married $90 \%, 5 \%$ as de facto relationship, and $5 \%$ as single. All of the mothers were living in Australia, with $21 \%$ of the sample reporting they lived in an urban setting, $73 \%$ in a regional setting, and $5 \%$ in a rural setting. With regard to highest educational level of the participating mothers, $26 \%$ of the mothers reported their highest education as secondary/high school education accounted, 57\% tertiary qualification/university degree, and $16 \%$ completed postgraduate qualifications. The mode household income was between $\$ 60,001$ and $\$ 80,000$ AUD.

Almost half the sample (47\%) had been attending the swim program for more than 12 months with $73 \%$ of the sample attending at least 3 sessions of the possible 6 per fortnight. Only $5 \%$ of the mothers attended an additional exercise program, swimming or otherwise, specifically for mothers. None of the sample reported being at their target level of fitness, $79 \%$ reported not being at their target weight, and $84 \%$ considered that they were not in their target physical shape. Prior to pregnancy $79 \%$ of the mothers reported exercising at least 3 or 4 times a week. During pregnancy 53\% reported exercising at least 3 or 4 times a week, and since becoming a mother $89 \%$ reported exercising at least 3 or 4 times a week. It should be noted that this was a purposeful biased sample towards exercise behavior with over half of the sample reporting that they changed their dietary and exercise habits since joining the swim program.

\section{Qualitative interview findings}

Preliminary analysis of the interviews revealed that the mothers' perceptions of what factors supported their adherence to the swim program were inherently intertwined with factors they considered positively influenced their mental health. For example, many mothers highlighted enjoyment of the program as a key factor in facilitating their continued participation as well as positively affecting their psychological wellbeing. Furthermore, numerous mothers highlighted improved and maintained mental health as an outcome of participating in the program, but with this outcome also being a motive to participate in the program. Because of this interconnectedness between antecedents and outcomes of the program, interview data were analyzed simultaneously to determine mothers' perception of factors related to program adherence and psychological wellbeing.

Two hundred and eighty-one (281) statements were grouped into 25 initial codes that were classified into 9 themes, and then further collapsed into 3 higher order themes (see Figure 2). During the interviews only one mother spoke of her like of swimming. The three higher order themes, environmental, social support, and health promoting, were considered to represent factors of the program that supported mothers' adherence (i.e. motivated and enabled them to continue to attend) as well as factors that represented how the program supported positive psychological wellbeing outcomes.

\section{Environmental factors}

The organization of the program, the coaches' styles, the onsite childcare facilities, and the physical environment of the swim program were all seen as important. The training program being set by someone else other than by the mother herself was a consistent relief for the interviewed mothers: "not having to think about what swim to do is such a relief from having to think about everything... a nice relief"; "being given a session takes a mental load-off". The coaches' ability to balance a fun and enjoyable approach with a structured and progressive training program that pushed the mothers was clearly seen as very important. The coaches' roles in providing critical yet supportive feedback and technical advice was integral in facilitating the mothers' perceived achievement and facilitated a sense of progression and success regarding their swimming and fitness ability.

The provision of onsite childcare was cited by the majority of the mothers as being pivotal in enabling them to attend the exercise program: "having the crèche (here) makes my swim possible", "it's import to me that I feel my children are safe while I'm working-out", "the way the place is setup is great, knowing that if there is a problem (with my child) that they (the crèche staff) can just come and tap me on the head at the end of a lap so I can jump out is very comforting to me". 


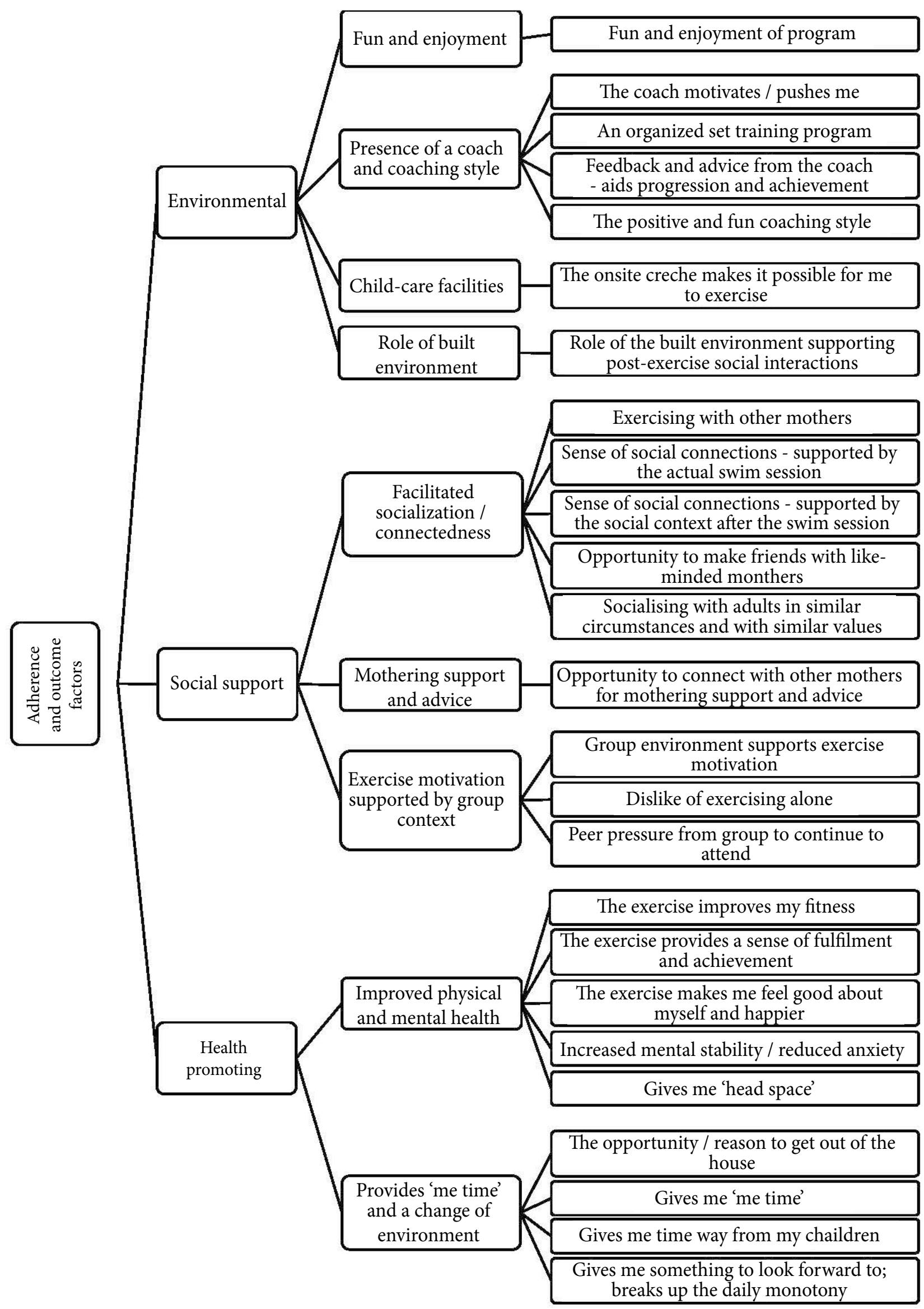

Figure 2: Factors supporting adherence and positive psychological wellbeing: Higher order themes, sub-themes, and initial codes.

The physical environment of the program, i.e. the provision of pleasant environment to socialize after the swim session (generally between 15 and 45 minutes) and connect with other mothers (usually accompanied 
by a cup of coffee) was clearly a major contributor to the program's success: "It gives me space from my little darling. Here I can do my session. I love hanging out here too, kids love playing too".

\section{Social support}

Substantially enabled by the aquatic center coffee shop and linked to the physical environment theme above, many of the mothers commented upon how their sense of connectedness with other mothers was facilitated by the access and opportunity of social congregations after each session while still at the aquatic center where children could also play: "I definitely use my coffee and chat after as my motivation and reward for swimming". For many of the mothers this was one of their most significant opportunities to socialize with other adults, especially with likeminded mothers of similar values and interests (i.e. exercise and health life styles): "I joined the squad for fitness, trying to get back in shape after baby. I still enjoy the fitness aspect but I really treasure the friendships I have made here... you know, doing something positive for me with other women with similar values". Many commented upon how other mothers' social gatherings did not lead to such enduring friendships as they frequently perceived that they did not have anything in common with mothers at other gatherings bar simply being a mother, "I tried other local groups when I first came to the coast. I felt like I just didn't fit in, it's different here, at least we're all trying to be healthy and do the right thing. I just feel more at home with these mums". While the social gathering post-exercise clearly played a key role in fostering the mothers' sense of connectedness, the actual swim sessions also provided a sense of community due to the group nature of the sessions and their enjoyment in the activity. This also links to the reported friendly banter between the mothers encouraged by the coach during the rest periods between sets. Mothers also indicated that being part of the group supported their exercise behaviors by providing additional motivation to attend and exercise: "the sessions are fun, the joking and friendly competition between sets makes the session pass quickly", "the coach does well in try to control us all and keep us on track; she balances the fun and jokes with making a good work-out that does push me".

The high level of socialization and perceived connectedness between the mothers facilitated by the group based exercise context, was associated with a significant exchange of mothering related information, e.g., approaches to weaning, how to get baby to sleep through the night, treatment of minor child illness, as well as general comradely support: "it's also a safe place to ask for useful advice on mothering challenges, you know, getting baby to sleep through the night, what to feed them, what are the best nurseries and schools", "everyone seems to have advice to give on the best way to look after your children, but with this group you feel that they, mostly, have similar values and lifestyles, wanting to give their children the healthiest start possible, it's been really useful to me here".

\section{Health promotion}

Not only did many of the mothers comments upon how the program helped them improve their physical fitness and appearance (actual and perceived), but also how the program improved their psychological wellbeing. For many the outcome of better psychological wellbeing associated with completion of the exercise session was a clear motive to continue attending the swim program. Several mothers referred to the exercise as their weekly "meds" and attitude adjustment: "it's this or wine to keep me sane!", "if I get here a couple of times a week I can really feel it, certainly makes me less up tight, my husband notices too".

Another common theme from the mothers was that the swim program gave them the motivation and opportunity to get out of the house along with a break from the children. This "me time" was also associated with something to look forward to that broke the potentially otherwise monotony of motherhood related childcare and household chores: "I love my baby, but this mummy time", "having that space for me, knowing the crèche is right there, is really important for me", "getting round to doing something for me with everything else going on is tough, but this make it easier, I have to be here at 9:15 because that's when it starts, so I get here at 9:15. If it was just me, I won't get round to fitting it, then I feel like the day's got away from me and I didn't really achieve much, just got through the day".

Again linking back to the presence of a coach, mothers also explained how the coaches' individual feedback frequently facilitated a sense of achievement and personal fulfillment, "not having a focus on work right now, because of child raising responsibilities, having a goal to work towards here in the pool, for me, I find is really important, gives me something to think about more than just being a mum", "I was never that good at sports at school, but I feel that I'm making progress here".

\section{Discussion}

The aim of this investigation was to examine what aspects of a mother-focused exercise program (swimming) that participating mothers considered most influential in supporting their continuing exercise adherence, as well as identifying what factors of the swim exercise program they felt positively influenced their psychological wellbeing. The first finding from this research was that the mothers' perceptions of the factors supporting their adherence to the swim program were inherently intertwined with factors they considered to positively influence their mental health.

Thematic content analysis of the interviews regarding the mothers' experiences of the swim program highlight- 
ed three higher order themes associated with supporting continued participation and positive psychological outcomes: Environmental factors; social support factors; and health promoting factors. These three factors do appear to fit with the SDT [24-26] and BNT [27]. In combination these theories attempt to explain motivation and psychological wellbeing. The environmental factor and its sub-themes aligned with satisfaction of each of the basic psychological needs. The sub-themes of fun and enjoyment, and role of built environment, aligned with relatedness satisfaction. The sub-theme, presence of a coach and coaching style, associated with sense of progression and achievement facilitated by the coaches' feedback related to competence satisfaction. The child-care facilities sub theme, enabled autonomy satisfaction. Clearly the social support factor highlighted by our results aligns with satisfaction of the mothers' psychological need for relatedness. The health promotion theme aligned with both competency and autonomy needs satisfaction. The subtheme of improved physical and mental health, related to perceptions of competency. While the subtheme of attainment of 'me time', a change in environment, doing something for herself away from her child/ren, for the benefit of herself, aligns with autonomy need satisfaction. These findings do provide further support for application of SDT and BNT to mothers' exercise contexts posited by Lovell, et al. [29].

\section{Conclusion}

The major implication of our findings is for the efficacious design of programs to increase mothers' exercise incidence and enhance psychological outcomes of such programs. Basing exercise programs around the environmental, social support, and health promotion themes highlighted by our research should help support positive outcomes. The observation that only one mother spoke of her enjoyment of swimming during the interviews has important implications for these findings. It appears that the success of the program was not directly related to the actual activity per se, but more so environmental, social support, and health promoting factors. These findings of what the important ingredients for a successful mothers exercise program to enhance participation and wellbeing should be generalizable to the constructing of effective interventions and programs based around any sport.

While this investigation has theoretical and applied implications, it should also be noted that this study does has limitations. The major limitation is the potential to which these findings and conclusions may be generalizable to mothers of other demographic contexts; particularly to those mothers from less advantaged socioeconomic status. Further study should consider these potentially important social and demographic issues.

\section{References}

1. Cutrona CE, Troutman B (1986) Social support, infant temperament, and parenting self-efficacy: A mediational model of postpartum depression. Child Dev 57: 1507-1518.
2. Dalton K (1980) Depression after childbirth. Oxford: Oxford University Press.

3. Gregoire A (1995) Hormones and postnatal depression. $\mathrm{Br}$ J Midwifery 3: 99-103.

4. O'Hara MW, Swain AM (1996) Rates and risk of postnatal depression - A meta-analysis. Int Rev Psychiatry 8: 37-54.

5. Paykel ES, Cooper Z (1992) Life events and social stress. In: Paykel ES, Handbook of affective disorders. Guilford Press, New York, 149-170.

6. Lovell GP, Butler FR (2015) Physical activity behaviour and role overload in mothers. Health Care for Women Int 36: 342-355.

7. Gore SA, Brown DM, West DS (2003) The role of postpartum weight retention in obesity among women: A review of the evidence. Ann Behav Med 26: 149-159.

8. Mindell JA, Jacobson BJ (2000) Sleep disturbances during pregnancy. J Obstet Gynecol Neonatal Nurs 29: 590-597.

9. Dritsa M, Da Costa D, Dupuis G, Lowensteyn I, Khalifé S (2008) Effects of a home-based exercise intervention on fatigue in postpartum depressed women: Results of a randomized controlled trial. Ann Behav Med 35: 179-187.

10. Mottola MF, Campbell MK (2003) Activity patterns during pregnancy. Can J Appl Physiol 28: 642-653.

11. Lewis BA, Kennedy BF (2011) Effects of exercise on depression during pregnancy and postpartum: A review. Am J Lifestyle Med 5: 370-378.

12. Davis K, Dimidjian S (2012) The relationship between physical activity and mood across the perinatal period: A review of naturalistic and clinical research to guide future investigation of physical activity-based interventions for perinatal depression. Clin Psychol Sci and Pract 19: 27-48.

13. Ekkekakis P, Backhouse SH (2009) Exercise and psychological well-being. In: Maughan RJ, Olympic Textbook of Science in Sport. Oxford: Wiley-Blackwell, 251-271.

14. Biddle S, Mutrie N (2008) Physical activity: A feel-good effect? In: Biddle S, Mutrie N, Psychology of physical activity: Determinants, well-being and interventions. Oxon: Routledge, 161-284.

15. Daley A, Jolly K, MacArthur C (2009) The effectiveness of exercise in the management of post-natal depression: Systematic review and meta-analysis. Fam Pract 26: 154-162.

16. Lovell GP, Huntsman A, Hedley-Ward J (2015) Psychological distress, depression, anxiety, stress, and exercise in Australian and New Zealand mothers: A cross-sectional survey. Nursing and Health Sciences 17: 42-48.

17. Bellows-Riecken KH, Rhodes RE (2008) A birth of inactivity? A review of physical activity and parenthood. Prev Med 46: 99-110.

18. Brown WJ, Mishra G, Lee C, Bauman A (2000) Leisure time physical activity in Australian women: Relationship with well-being and symptoms. Res Quart Exerc Sport 71: 206-216.

19. Ansari W, Lovell GP (2009) Barriers to exercise in younger and older non-exercising adult women: A cross sectional study in the United Kingdom. Int J Environ Res Public Health 6: 1443-1455.

20. Albright C, Maddock JE, Nigg CR (2005) Physical activity before pregnancy and following childbirth in a multiethnic sample of healthy women in Hawaii. Women Health 42: 95-110.

21. Currie JL, Develin ED (2002) Stroll your way to well-being: A survey of the perceived benefits, barriers, community 
support, and stigma associated with pram walking groups designed for new mothers, Sydney, Australia. Health Care Women Int 23: 882-893.

22. King AC, Castro C, Wilcox S, Eyler AA, Sallis JF, et al. (2000) Personal and environmental factors associated with physical inactivity among different racial-ethnic groups of US middle-aged and older-aged women. Health Psychol 19: 354-364.

23. Armstrong K, Edwards $\mathrm{H}$ (2004) The effectiveness of a pram-walking exercise programme in reducing depressive symptomatology for postnatal women. Int J Nurs Pract 10 : 177-194.

24. Deci EL, Ryan RM (1985) Intrinsic motivation and self-determination in human behavior. Plenum Press, New York.

25. Deci EL, Ryan RM (2000) The "what" and "why" of goal pursuits: Human needs and the self-determination of behavior. Psychol Inq 11: 227-268.
26. Deci E, Ryan RM (2002) Handbook of self-determination research. The University of Rochester Press, Rochester.

27. Ryan RM, Deci EL (2000) Self-determination theory and the facilitation of intrinsic motivation, social development, and wellbeing. Am Psychol 55: 68-78.

28. Josefsson KW (2016) Mechanisms in self-determined exercise motivation. A PhD thesis summary. Int $\mathrm{J}$ Womens Health Wellness 2: 033.

29. Lovell GP, Gordon JA, Mueller MB, Mulgrew K, Sharman R (2016) Satisfaction of basic psychological needs, self-determined exercise motivation, and psychological wellbeing in mothers exercising in group-based versus individual-based contexts. Health Care Women Int 37: 568-582.

30. Braun V, Clarke V (2006) Using thematic analysis in psychology. Qual Res Psychol 3: 77-101. 